\title{
Dihydroartemisinin Prevents Progression and Metastasis of Head and Neck Squamous Cell Carcinoma by Inhibiting Polarization of Macrophages in Tumor Microenvironment
}

\author{
This article was published in the following Dove Press journal: \\ OncoTargets and Therapy
}

\author{
Ran Chen ${ }^{1,2}$ \\ Xiuying $\mathrm{Lu}^{2}$ \\ Zhen $\mathrm{Li}^{2}$ \\ Yajing Sun ${ }^{2}$ \\ Zhengxin $\mathrm{He}^{3}$ \\ Xiaoming $\mathrm{Li}^{1,2}$ \\ 'Graduate School of Hebei Medical \\ University, Shijiazhuang, People's Republic \\ of China; ${ }^{2}$ Department of \\ Otolaryngology Head and Neck Surgery, \\ The 980th Hospital of PLA Joint Logistics \\ Support Force, Shijiazhuang, People's \\ Republic of China; ${ }^{3}$ Department of \\ Laboratory Medicine, The 980th Hospital \\ of PLA Joint Logistics Support Force, \\ Shijiazhuang, People's Republic of China
}

Background: Polarized M2 macrophages are an important type of tumor-associated macrophage (TAM), with roles in the growth, invasion, and migration of cancer cells in the tumor microenvironment. Dihydroartemisinin (DHA), a traditional Chinese medicine extract, has been shown to inhibit the progression and metastasis of head and neck squamous cell carcinoma (HNSCC); however, the effect of DHA on cancer prevention, and the associated mechanism, has not been investigated in the tumor microenvironment.

Materials and Methods: First, human Thp-1 monocytes were induced and differentiated into M2 macrophages using phorbol 12-myristate 13-acetate (PMA), interleukin-6 (IL-6), and interleukin-4 (IL-4). Induction success was confirmed by cell morphology evaluation, flow cytometry, and quantitative real-time polymerase chain reaction (qRT-PCR). Then, DHA was applied to interfere with M2 macrophage polarization, and conditioned medium (CM), including conditioned medium from M2 macrophages (M2-CM) and conditioned medium from M2 macrophages with DHA (M2-DHA-CM), was obtained. CM was applied to Fadu or Cal-27 cells, and its effects on cancer invasion, migration, and angiogenesis were evaluated using transwell, wound-healing, and tube formation assays, respectively. Finally, Western blotting was used to evaluate the relationship between signal transducer and activator of transcription 3 (STAT3) signaling pathway activation and M2 macrophage polarization.

Results: Human Thp-1 monocytes were successfully polarized into M2-like TAMs using PMA, IL-6, and IL-4. We found that M2-like TAMs promoted the invasion, migration, and angiogenesis of HNSCC cells; however, DHA significantly inhibited IL-4/IL-6-induced M2 macrophage polarization. Additionally, as DHA induced a decrease in the number of M2-like TAMs, M2-DHA-CM inhibited the induction of invasion, migration, and angiogenesis of Fadu and Cal-27 cells. Finally, DHA inhibited M2 macrophage polarization by blocking STAT3 pathway activation in macrophages.

Conclusion: DHA inhibits the invasion, migration, and angiogenesis of HNSCC by preventing M2 macrophage polarization via blocking STAT3 phosphorylation.

Keywords: dihydroartemisinin, tumor-associated macrophages, HNSCC, STAT3, macrophage polarization

\section{Introduction}

Head and neck squamous cell carcinoma (HNSCC) is a type of cancer that occurs in the oral cavity, nasal cavity, pharynx, and larynx. It ranks as the sixth most aggressive malignancy worldwide, with a five-year survival rate of only
Correspondence: Xiaoming Li Department of Otolaryngology Head and Neck Surgery, Hebei Medicial University, Shijiazhuang 050017, People's Republic of China, The 980th Hospital of PLA Joint Logistics Support Force, Shijiazhuang 050082, People's Republic of China

$\mathrm{Tel} / \mathrm{Fax}+8631 \mathrm{I} 87978418$

Emailxmlmo@I26.com 
approximately $50 \% .^{1-3}$ More than $90 \%$ of HNSCC patients die, not from the rupture and bleeding of their primary cancer, but rather from cancer metastases. ${ }^{4}$ The continued growth, invasion, and migration of cancer depends, not only on genetic mutations in cancer cells but also on the support and regulation of the tumor microenvironment. Therefore, exploration of the relationship between the tumor microenvironment and the tumor could help to identify novel cancer treatments.

The tumor microenvironment comprises endothelial, immune, tumor, and other cells, which interact in complex ways in the tumor microenvironment. ${ }^{5,6}$ Tumor-associated macrophages (TAMs) are an important type of immune cell, and their roles in the tumor microenvironment are mainly determined by their M1 or M2 phenotype. ${ }^{7}$ Recent studies on oral and colon cancers have shown that M2-like TAMs can promote cell matrix decomposition, cancer cell migration, and blood and lymphatic vessel formation, all of which are necessary for tumor metastasis. ${ }^{8-10}$ Clinical studies of glioblastoma and oral squamous cell carcinoma have also shown that the presence of large numbers of M2-like TAMs is positively correlated with poor prognosis; therefore, M2-like TAMs represent a new therapeutic target, and inhibition of the polarization of M2 macrophages is an effective approach. ${ }^{1-13}$

Dihydroartemisinin (DHA) is a semisynthetic derivative extracted from Artemisia annua using modern technology and has antimalarial, anti-inflammatory, and anti-tumor effects. ${ }^{14-17}$ DHA has significant effects on various human tumors, including hepatocellular carcinoma and ovarian cancer; however, whether DHA can inhibit cancer progression and metastasis by regulating TAMs in the tumor microenvironment has not been reported. ${ }^{16,17}$ Therefore, we investigated the relationship between DHA and macrophage polarization in the tumor microenvironment using in vitro experiments. Our data demonstrate that DHA inhibits the invasion, migration, and angiogenesis of HNSCC by blocking the phosphorylation of STAT3 to prevent M2 macrophage polarization.

\section{Materials and Methods}

\section{Cell Culture and Drugs}

The HNSCC cell line Fadu, established a hypopharyngeal tumor from an Indian individual, was purchased from the Institute of Biochemistry and Cell Biology, Chinese Academy of Sciences (Shanghai, China). Human Thp-1 monocytes, derived from peripheral blood of a 1-year-old boy, were purchased from the Institute of Biochemistry and Cell Biology, Chinese Academy of Sciences (Shanghai, China). The Cal-27 cell line, established from a primary tongue cancer, was donated by the Institute of Stomatology, Nanjing Medical University, and purchased from American Type Culture Collection (ATCC; Manassas, USA). The human umbilical vein endothelial cell line (HUVECs) was donated by Dr. Hong Ji of Fudan University and purchased from ATCC (Manassas, USA). Fadu and Cal-27 cells were cultured in DMEM, while HUVECs were maintained in M199 medium (Solarbio, Beijing, China). Thp-1 cells were cultured in RPMI-1640 medium (Solarbio, Beijing, China). All cultures were supplemented with $10 \%$ fetal bovine serum (Gibco, Rockville, MD) and $1 \%$ penicillin and streptomycin (Gibco), and maintained at $37^{\circ} \mathrm{C}$ in $5 \% \mathrm{CO}_{2}$. DHA was purchased from Tokyo Chemical Industry (Tokyo, Japan).

\section{Polarization and Drug Treatment of Macrophages}

As shown in Figure 1B, to obtain M0 macrophages, $200 \mathrm{ng} /$ $\mathrm{mL}$ phorbol 12-myristate 13-acetate (PMA; Sigma, MO, USA) were added to Thp-1 monocytes, cells cultured for $24 \mathrm{~h}$, and then $20 \mathrm{ng} / \mathrm{mL}$ IL- 6 and IL-4 added to stimulate the cells for $24 \mathrm{~h}$ to obtain M2 macrophages. Cells were divided into four groups, according to the culture media used, as follows: M0, M0DHA, M2, and M2DHA. During M0 induction, suspended Thp- 1 cells began to become adherent after 8 to $12 \mathrm{~h}$; hence, $50 \mu \mathrm{M}$ DHA was added at $12 \mathrm{~h}$ in the M0DHA group. In the M2DHA group, DHA was added, along with IL-6 and IL-4 to obtain M2 macrophages.

\section{MTT Assays}

M0 monocytes were inoculated into 96-well plates at a density of $1 \times 10^{4} /$ well and different concentrations of DHA or dimethyl sulfoxide (DMSO) added. After $24 \mathrm{~h}$ of treatment, $10 \mu \mathrm{L}$ freshly prepared 3-(4,5-dimethylthiazol2-yl)-2,5-diphenyltetrazolium bromide (MTT) reagent was added to each well and the cells incubated at $37^{\circ} \mathrm{C}$ for 4 h. Then, $100 \mu \mathrm{L}$ of DMSO was added to each well and oscillated on a shaker at a low speed for $10 \mathrm{~min}$, room temperature. The absorbance value (OD, $490 \mathrm{~nm}$ ) of each well was measured using an enzyme-linked immunodetector (Model 550, Bio-Rad, Hercules, USA).

\section{Flow Cytometry}

Cells were detached from a six-well plate by trypsinization and a cell suspension $\left(1 \times 10^{6} / \mathrm{mL}\right)$ prepared. An anti-CD163 
A

Thp-1

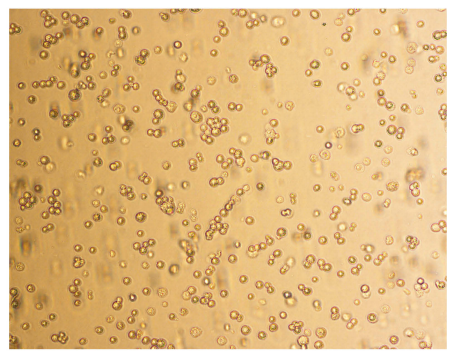

B

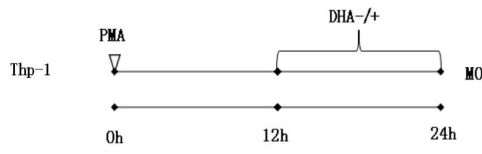

Thp-1

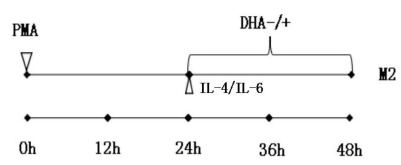

M0

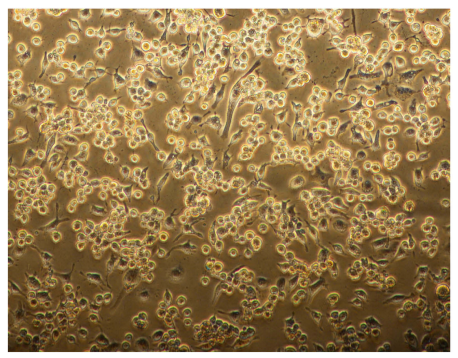

M2

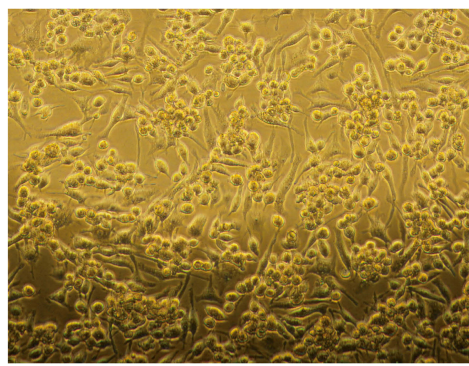

MODHA

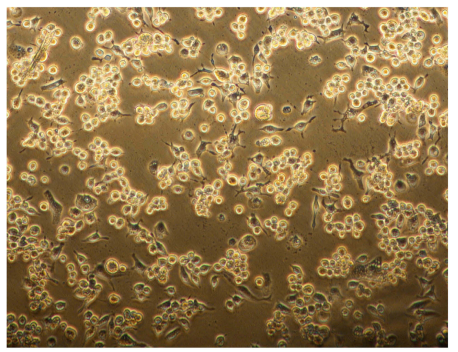

M2DHA

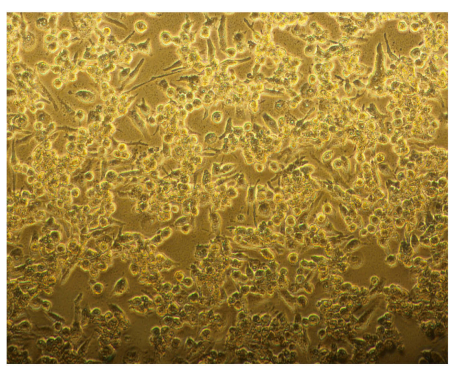

C
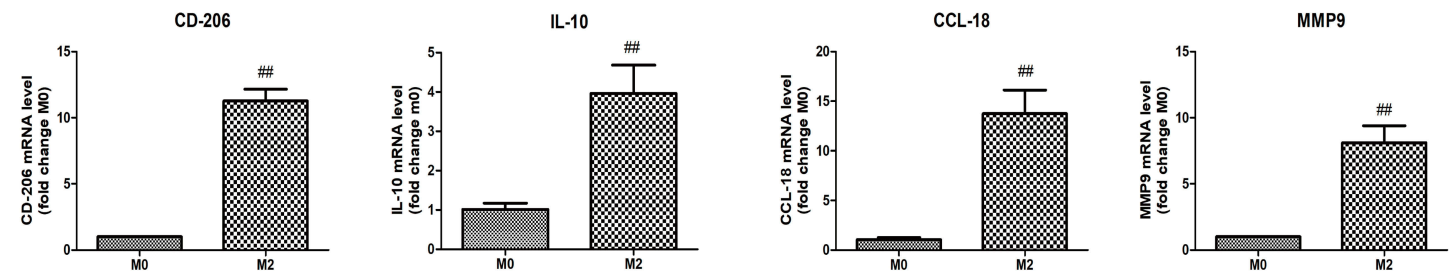

D
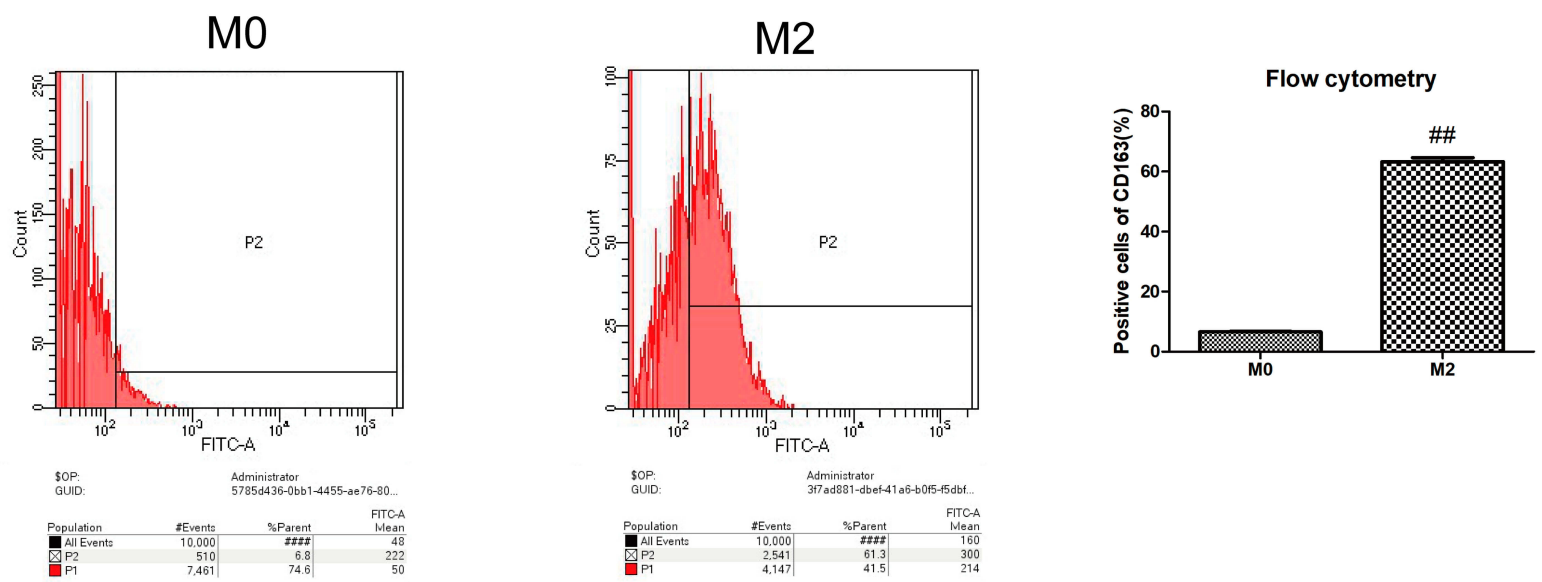

Figure I Thp-I cells were induced to become M2-like TAMs. (A) Thp-I cells were differentiated from macrophages (with or without drugs, polarized or unpolarized). (B)

A diagrammatic illustration for the M2 macrophage polarization and DHA treatment of Thp-I cells. (C) mRNA expression levels of some M0 and M2 genes differed. (D) Flow cytometry was used to determine the expression of M2 macrophage marker, CDI63.

Notes: Results are presented as mean \pm SD. \#\#p $<0.0$, compared with M0. 
antibody (1:60, Abcam Cambridge, UK) was added and incubated at room temperature for $30 \mathrm{~min}$. Then, the cells were washed three times and resuspended in $1 \mathrm{~mL}$ of phosphatebuffered saline (PBS). Cells were sorted using a FACSCalibur flow cytometer (BD Biosciences, USA) and analyzed with BD FACSDiva software (BD Biosciences, USA).

\section{Quantitative Real-Time PCR Assay (qRT-PCR)}

TRIzol reagent (Invitrogen, Carlsbad, USA) was used to extract total RNA from a variety of cells. Prime Script RT reagent kit (Takara, Dalian, China) was used for reverse transcription, and a TB green Premix Ex Taq (Takara, Dalian, China) was used for amplification. Glyceraldehyde3-phosphate dehydrogenase $(G A P D H)$ was used as an endogenous control for mRNA expression detection. Data were compared and analyzed using the $2^{-\Delta \Delta C t}$ method. All primer sequences are listed in Table 1.

\section{Preparation of Macrophage Medium}

Culture medium was removed from macrophages in different states (with or without drugs and polarized or unpolarized), and the cells washed three times with PBS, followed by the addition of serum-free RPMI-1640 medium for $24 \mathrm{~h}$. The obtained medium was centrifuged at $10,000 \mathrm{rpm}$ for $5 \mathrm{~min}$ and stored at $-80^{\circ} \mathrm{C}$ (hereafter referred to as macrophage conditioned medium (CM)).

\section{Enzyme-Linked Immunosorbent Assay (ELISA)}

Levels of the cytokine, interleukin-10 (IL-10), in conditioned medium from M0 macrophages with or without DHA treatment, conditioned medium from M2 macrophages with or without DHA treatment, conditioned medium from M0 with DMSO treatment, and conditioned medium from M2 macrophages with DMSO treatment were detected using a human IL-10 ELISA kit (BIKW BioCo, Ltd., Beijing, China) as per manufacture's instruction.

\section{Wounding-Healing Assay}

Fadu/Cal-27 cells were inoculated into 6-well plates at a density of $8 \times 10^{5} /$ well. The next day, a vertical scratch was made in the center of the cells using a pipette tip (200 $\mu \mathrm{L}$ ), and the detached cells were washed away with PBS. Each of the four kinds of macrophage CM or RPMI-1640 medium was mixed 1:1 with complete DMEM and then added to the 6-well plates. After $24 \mathrm{~h}$ in an incubator, cells were photographed using an inverted electron microscope (Olympus, Tokyo, Japan) with a digital camera (Canon, Tokyo, Japan) and analyzed using ImageJ software (National Institutes of Health, MD, USA).

\section{Transwell Invasion Assay}

A transwell chamber coated with $40 \mu \mathrm{L}$ Matrigel (Corning, NY, USA) at a concentration of $200 \mu \mathrm{g} / \mathrm{mL}$, was placed in a 24-well plate, and Fadu/Cal-27 cells inoculated at a density of $5.0 \times 10^{5} / \mathrm{mL}$. Each of four kinds of macrophage medium or RPMI-1640 medium was mixed 1:1 with complete DMEM and then added into the low chambers of the culture plate. After 48 $h$ cells were washed with PBS, fixed in paraformaldehyde for $20 \mathrm{~min}$, and dyed with crystal violet. A visual field was randomly selected under a microscope and analyzed using ImageJ software.

\section{Tube Formation Assay}

1. Each of four kinds of CM was mixed 1:1 with M199 complete medium and then stored for use. Matrigel $(70 \mu \mathrm{L}, 8 \mathrm{mg} / \mathrm{mL})$, was plated in 96-well plates and placed in an incubator for $30 \mathrm{~min}$, followed by addition of the medium and $1.5 \times 10^{4}$ /well HUVECs. After $6 \mathrm{~h}$, three fields were randomly observed at $200 \times$ magnification, and the lumens of formed tubes

Table I qRT-PCR Primers Used in This Paper

\begin{tabular}{|l|l|l|}
\hline Gene & Forward Primers & Reverse Primers \\
\hline VEGFA & AGGGCAGAATCATCACGAAGT & AGGGTCTCGATTGGATGGCA \\
MMP9 & AGACCTGGGCAGATTCCAAA & CGGCAAGTCTTCCGAGTAGT \\
MMPI0 & TTTGGCTCATGCCTACCCAC & TCTTGCGAAAGGCGGAACTG \\
CD206 & CAGGTGTGGGCTCAGGTAGT & TGTGGTGAGCTGAAAGGTGA \\
IL-10 & GGTTGCCAAGCCTTGTCTGA & AGGGAGTTCACATGCGCCT \\
CCLI8 & CTCTGCTGCCTCGTCTATACCT & CTTGGTTAGGAGGATGACACCT \\
GAPDH & TCCAAAATCAAGTGGGGCGA & AGTAGAGGCAGGGATGATGT \\
\hline
\end{tabular}


analyzed and cells counted using Axiovision Rel 4.1 software (Zeiss, Germany).

2. First, Fadu/Cal-27 cells were inoculated into 6-well plates at a density of $1 \times 10^{6} /$ well. Then, each of the four kinds of macrophage CM or RPMI-1640 medium was mixed 1:1 with complete DMEM and added to the 6-well plates. After $24 \mathrm{~h}$ in an incubator, the obtained medium was centrifuged at $10,000 \mathrm{rpm}$ for $5 \mathrm{~min}$ and stored at $-80^{\circ} \mathrm{C}$. Each of the four kinds of CM or RPMI-1640 medium was mixed 1:1 with M199 complete medium and then stored for use. Matrigel $(70 \mu \mathrm{L}, 8 \mathrm{mg} / \mathrm{mL})$, was plated in 96-well plates and placed in an incubator for $30 \mathrm{~min}$, then medium and $1.5 \times 10^{4} /$ well HUVECs added to the 96-well plate. After $6 \mathrm{~h}$, three fields were randomly observed at $200 \times$ magnification, and the lumens of formed tubes analyzed and cells counted using Axiovision Rel 4.1 software.

\section{Western Blot}

HNSCC cells and other cells from 6-well plates or tissue specimens were harvested in a lysis buffer (Beyotime Institute of Biotechnology, Haimen, China) and incubated for $30 \mathrm{~min}$ at $4^{\circ} \mathrm{C}$. Proteins were obtained after centrifugation at 13,000 rpm for $10 \mathrm{~min}$ and then quickly frozen. Equivalent amounts of proteins were separated by $10 \%$ sodium dodecyl sulfate-polyacrylamide gel electrophoresis (SDS-PAGE) and then transferred to polyvinylidene fluoride membranes (Millipore Corporation, Temecula, CA). After blocking with TBS plus 5\% nonfat milk for $3 \mathrm{~h}$ at room temperature, membranes were incubated with primary antibodies, including anti-STAT3 (1:2,000; Cell Signaling Technology, Boston, USA), anti-p-STAT3 (1:2,000; Cell Signaling Technology, Boston, USA), antiE-cadherin (1:1000; Proteintech Group Inc., Chicago, USA), anti-Vimentin (1:1000; Proteintech Group Inc., Chicago, USA), and anti-rabbit- $\beta$-actin $(1: 3,000$; Proteintech, Wuhan, China), overnight in a refrigerator. Membranes were then incubated with a secondary antibody (1:5,000; Golden Bridge Co., Ltd., Beijing, China) for $1 \mathrm{~h}$ at room temperature. After washing in TBST, membranes were incubated with SuperSignal ${ }^{\mathrm{TM}}$ West Dura Extended Duration Substrate (Thermo Fisher Scientific, Waltham, USA). Immunoreactive proteins were visualized using the Chemiluminescence Detection System (GE Healthcare Life Sciences, Amersham, UK) or Fusion FX5 Spectra (Vilber, French).

\section{Lentivirus Infection of Target Cells}

According to the manufacturer's suggestion (Hanbio, Shanghai, China), cells in good condition at a density of $3 \times 10^{5} /$ well were inoculated into 12 -well plates, treated with a multiplicity of infection of 50 using the lentivirus vectors, rlv-hstat3-hygro and rlv-luciferase-puro (Hanbio), and cultured overnight at $37^{\circ} \mathrm{C}$ in a $5 \% \mathrm{CO}_{2}$ incubator. After $24 \mathrm{~h}$, fresh complete RPMI-1640 medium was added for $24 \mathrm{~h}$. After the cells had been incubated with the lentiviruses for $48 \mathrm{~h}, 5 \mu \mathrm{g} / \mathrm{mL}$ puromycin and $500 \mu \mathrm{g} / \mathrm{mL}$ hygromycin (Hanbio) were used for drug screening. Finally, p-STAT3 expression was detected by Western blotting.

\section{Statistical Analysis}

Data are expressed as the mean $\pm \mathrm{SD}$. Student's $t$-test or one-way ANOVA were used for statistical analyses, as appropriate. All results were considered statistically significant at $\mathrm{p}<0.05$. All graphs were plotted using GraphPad Prism 7 (GraphPad Software Inc, CA, USA).

\section{Results}

\section{Thp-I Cells Were Induced to Become M2-Like TAMs}

As shown in Figure 1, stimulation with PMA induced Thp1 cells to become macrophages; cell morphology changed from suspension cells to adherent cells with obvious pseudopodia (Figure 1A). After continuous stimulation with PMA and IL-4/IL-6, the expression of CD163, a surface marker of M2-like TAMs, increased significantly (Figure 1D). As expected, levels of IL-10, CD206, matrix metalloproteinase-9 (MMP-9), and chemokine ligand-18 (CCL18) mRNA were upregulated with the increase in CD163 (Figure 1C). IL-10 and CD206 expression levels were in direct proportion to the number of M2 macrophages. ${ }^{18,19}$ These data indicate that Thp-1 monocytes were successfully polarized into M2-like macrophages using PMA, IL6 , and IL-4.

\section{DHA Inhibited M2-Like TAM Polarization in vitro}

To test whether DHA can inhibit M2-like TAM polarization, we first determined the appropriate concentration of DHA. A toxicity study showed that DHA and DMSO did not kill macrophages (Figure 2A). Based on previous MTT results for the Fadu and Cal-27 cell lines generated in our laboratory, the concentration of DHA was set at $50 \mu \mathrm{M} .{ }^{20}$ We used the levels of the M2-like TAM surface marker, CD163, to 
A

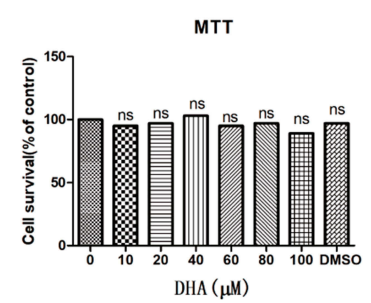

MO

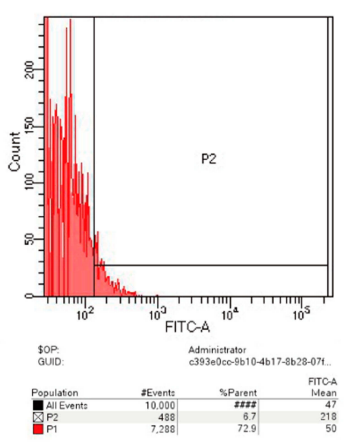

D

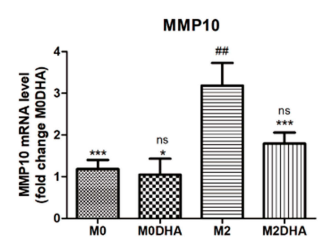

M0

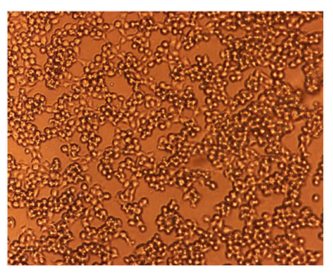

B

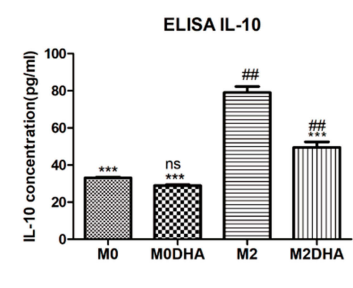

MODHA
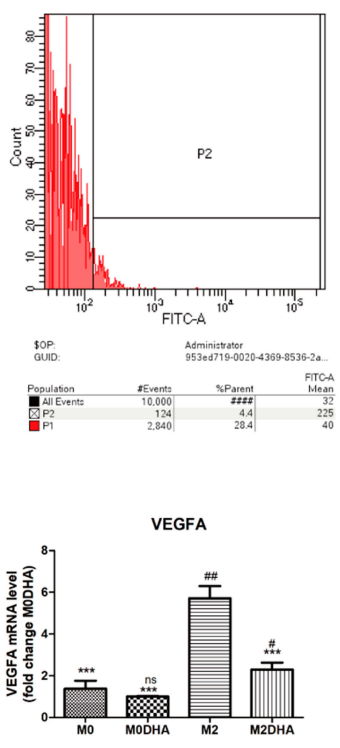

MODHA

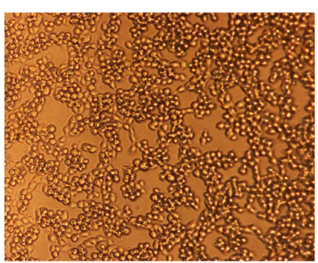

C
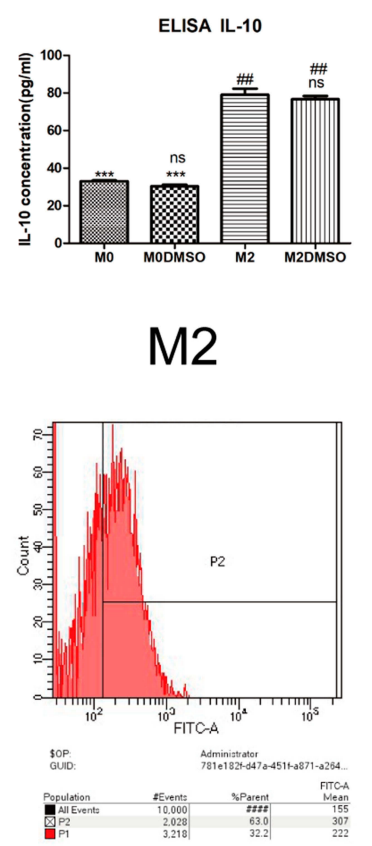

E

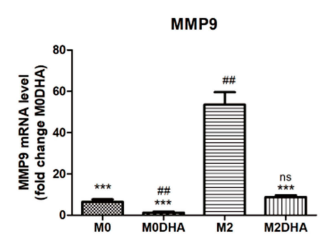

M2

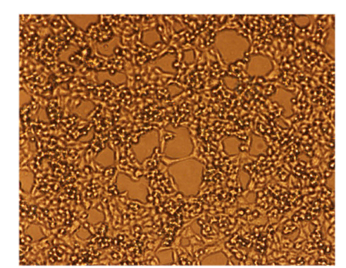

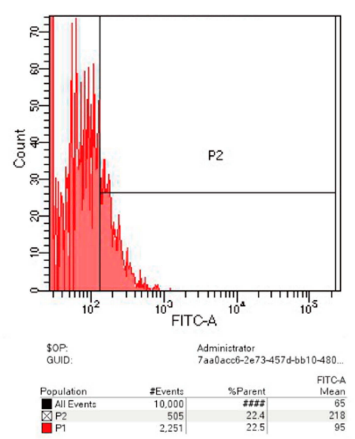

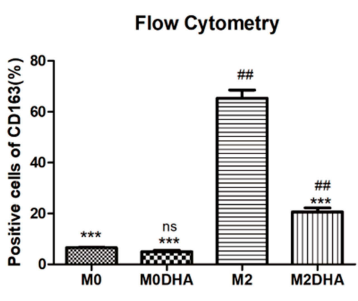

M2DHA

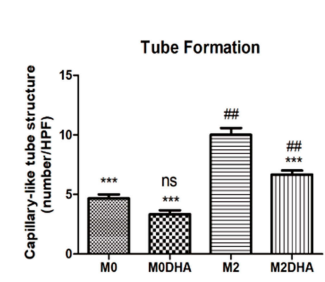

M2DHA

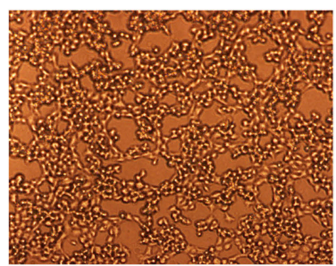

Figure 2 DHA inhibited M2-like TAM polarization in vitro. (A) MTT was used to evaluate the toxicity of DHA and DMSO against macrophages. (B) Levels of IL-I0 in the cell culture medium were measured by ELISA. (C) Flow cytometry was used to evaluate the expression of the M2 macrophage marker, CDI63. (D) qRT-PCR was used to detect the expression levels of CD206, MMP9, MMPI0, and VEGFA genes in M2 macrophages. (E) The tube formation assay was used to examine the effect of conditioned medium on angiogenesis.

Notes: Results are presented as mean $\pm \mathrm{SD} .{ }^{\#} \mathrm{P}<0.0 \mathrm{I},{ }^{\#} \mathrm{p}<0.05$, ns $=$ not significant, compared with $\mathrm{M0}$; ${ }^{* * *} \mathrm{p}<0.0 \mathrm{I},{ }^{*} \mathrm{p}<0.05$, ns $=$ not significant, compared with $\mathrm{M} 2$.

represent the number of M2-like TAMs. As shown in Figure $2 \mathrm{C}$, the number of M2-like TAMs clearly increased significantly with the addition of IL-4 and IL-6, while addition of DHA led to a significant decrease in the number of M2-like TAMs. ELISA results also demonstrated a reduction in IL-10 expression on addition of DHA (Figure 2B). Evaluation of mRNA expression further confirmed the effect of DHA, which significantly decreased the expression levels of vascular endothelial growth factor A (VEGFA), MMP-9, and matrix metalloproteinase-10 (MMP-10) (Figure 2D). Moreover, tube formation assays demonstrated that M2-like TAMs promoted vascular formation, which decreased with DHA treatment (Figure 2E). Given the expression levels of IL-10, determined by ELISA and MTT results (Figure 2A and $\mathrm{B}$ ), our results demonstrate that DMSO did not reduce the quantity of M2-like TAMs. 


\section{DHA Inhibited HNSCC Cells Invasion, Metastasis, and Angiogenesis by Inhibiting M2-Like TAM Polarization}

Previous studies have identified M2-like TAMs as contributing to the migration, invasion, and angiogenesis of various cancers, including breast, liver, and gynecological cancers. $^{20-22}$ To determine the relationship between M2like TAMs and HNSCC cells, as well as the effect of DHA, we conducted several experiments. Woundinghealing experiments and Western blot analysis showed that CM from M2-like TAMs significantly promoted HNCSS cells migration, while in the M2DHA group, CM decreased this effect (Figure 3A). A transwell invasion assay demonstrated that $\mathrm{M} 2-\mathrm{CM}$ had stronger invasionpromoting effects on Fadu and Cal-27 cells than M0-CM, while M2-DHA-CM significantly reduced tumor invasion (Figure 3B). In addition, Fadu and Cal-27 cells had an increased effect on tumor angiogenesis in response to M2like TAMs, and an inhibitory effect of DHA on angiogenesis was also detected (Figure 3C).

\section{DHA Inhibited M2-Like TAM Polarization by Inhibiting STAT3 Phosphorylation}

To explore the mechanism by which DHA inhibits M2-like TAM polarization, we made our conjecture based on the following findings: i) IL-6 can activate the STAT3 pathway, which is initiated by phosphorylation of the STAT3 protein $^{23}$ ii) DHA inhibits phosphorylation of the STAT3; ${ }^{24}$ iii) phosphorylation of the STAT3 is closely associated with differentiation of M2 macrophages. ${ }^{25,26}$ Therefore, we postulated that DHA could inhibit the M2like TAMs polarization by inhibiting STAT3 phosphorylation. Western blot analysis confirmed our hypothesis, as p-STAT3 levels were upregulated by IL-4/IL-6 stimulation in M2-like TAMs, and this effect was reduced by DHA. Moreover, STAT3 expression did not change in response to IL-4/IL-6 treatment, whereas p-STAT3 levels were elevated in a time-dependent manner; however, DHA inhibited this increase, particularly $24 \mathrm{~h}$ after its addition (Figure 4A). Lentiviruses were used to activate the phosphorylation of macrophage STAT3 and Western blot analysis showed that p-STAT3 levels were significantly higher in the lentivirus group than those in the control group (Figure 4B), while STAT3 protein levels were not increased. As shown in Figure 4C, flow cytometry results demonstrated that IL-4/IL-6 stimulation led to higher CD163 expression levels in the lentivirus-treated group relative to the control group; however, after DHA treatment, CD163 expression levels in the control group decreased more significantly. The data showed that the lentivirus continued to activate the STAT3 pathway and competitively prevented DHA inhibition of macrophage polarization (Figure 4C and D). These results indicate that activation of the STAT3 pathway is directly related to the inhibitory effect of DHA on M2-like TAM polarization.

\section{Discussion}

Surgery, radiotherapy, and chemotherapy are the cornerstones of head and neck cancer therapy; however, they all have many limitations and side effects in clinical practice. For chemoradiation therapy, it affects not only tumor cells but also normal cells of the body. To minimize the toxic and side effects caused by chemoradiation, we may need a new approach that targets some non-tumor cell populations in the tumor microenvironment, like TAMs.

With breakthroughs in our understanding of the tumor microenvironment, TAMs have become a focus of cancer research, as they can produce various specific chemokines and cytokines in the tumor microenvironment which are necessary for cancer growth and metastasis. ${ }^{18,27}$ TAMs can promote progression and metastasis of breast, ovarian, lung, gastric, endometrial, and other cancers. ${ }^{20-22,28-30}$ In HNSCC, TAMs are recruited into the tumor microenvironment and directly contact squamous cell carcinoma cells to promote cancer progression, metastasis, and angiogenesis. $^{31-33}$ M2-like TAMs are the predominant type of TAMs, and numbers are directly related to cancer angiogenesis, radiotherapy, and chemotherapy responses, as well as overall survival rate. ${ }^{34-38}$ Our in vitro experiments also showed that an increase or decrease in M2-like TAMs was positively associated with the invasion, migration, and angiogenesis of Fadu and Cal-27 cell lines. Hence, M2-like TAMs are the targets for treatment.

Currently, targeting treatments for TAMs are as follows: i) reduce the total number of TAMs or macrophages; ii) repolarize M2-like macrophages into M1-like macrophages; iii) reduce or inhibit tumor recruitment of TAMs; and iv) inhibit TAM activation, particularly of M2-like macrophages. Therefore, traditional Chinese medicine, particularly traditional Chinese medicine monomers, provides many promising options for TAM-targeting treatments. For example, resveratrol kills macrophages and reduces the production of blood vessels and lymphatic vessels in the tumor microenvironment. ${ }^{25}$ Hesperetin 
A

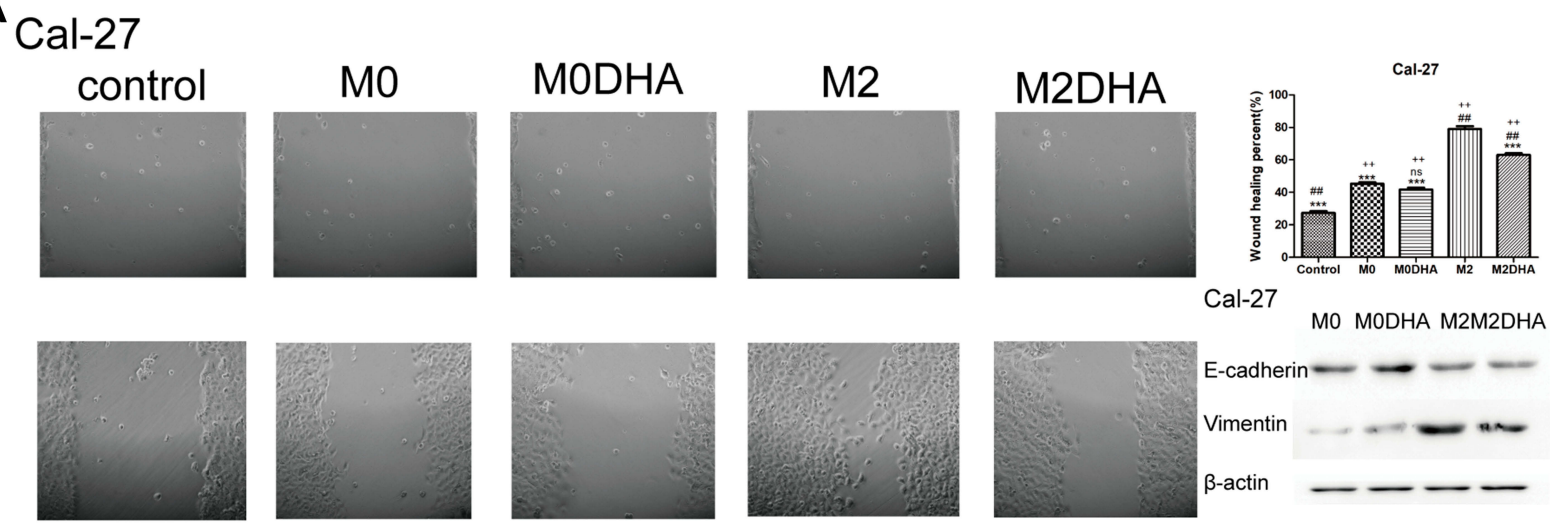

Fadu
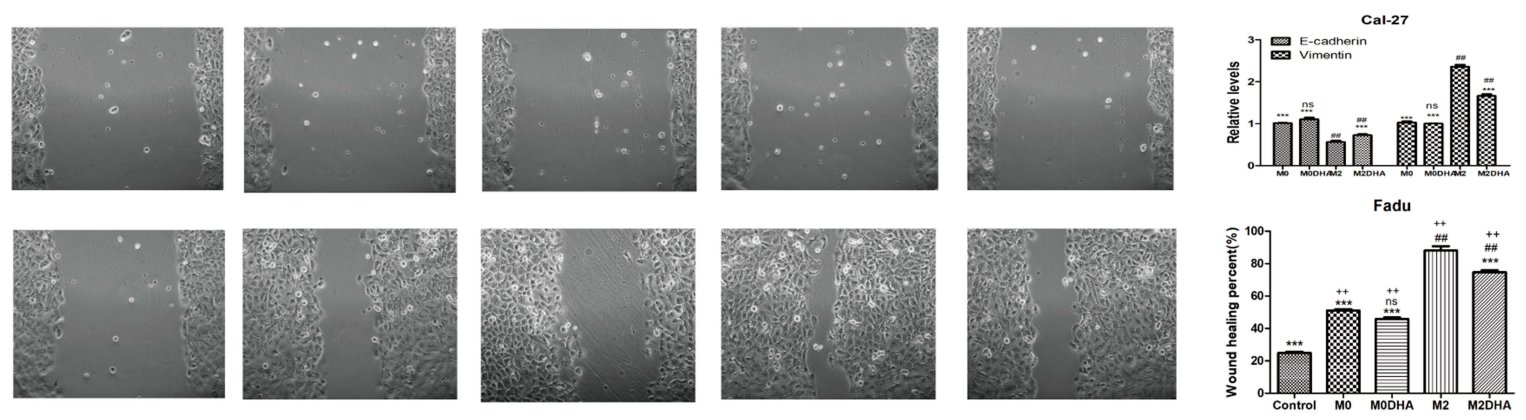

B

\section{Cal-27}
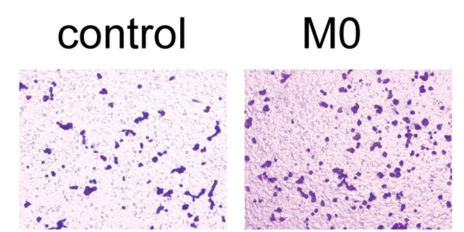

\section{MODHA}

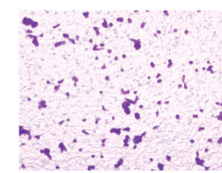

Fadu
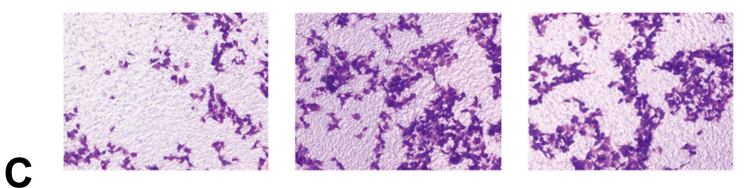

\section{Cal-27}
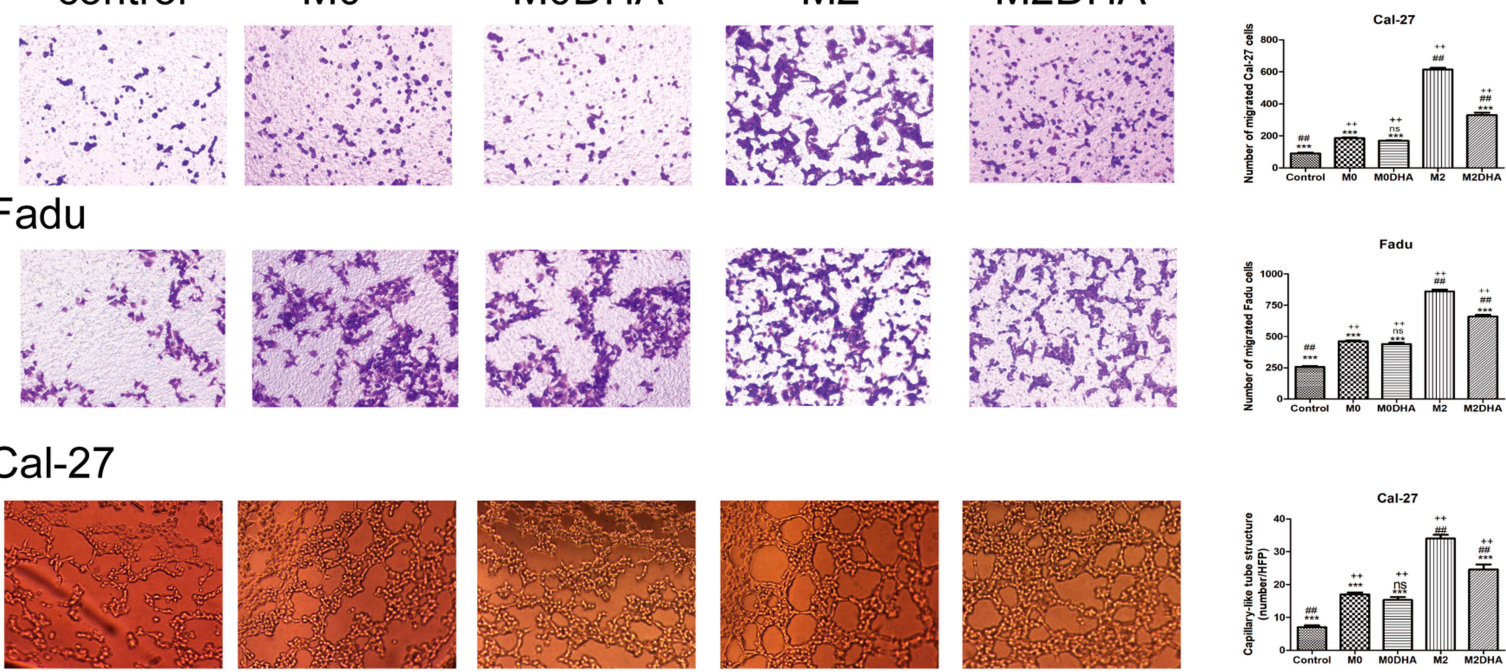

Fadu
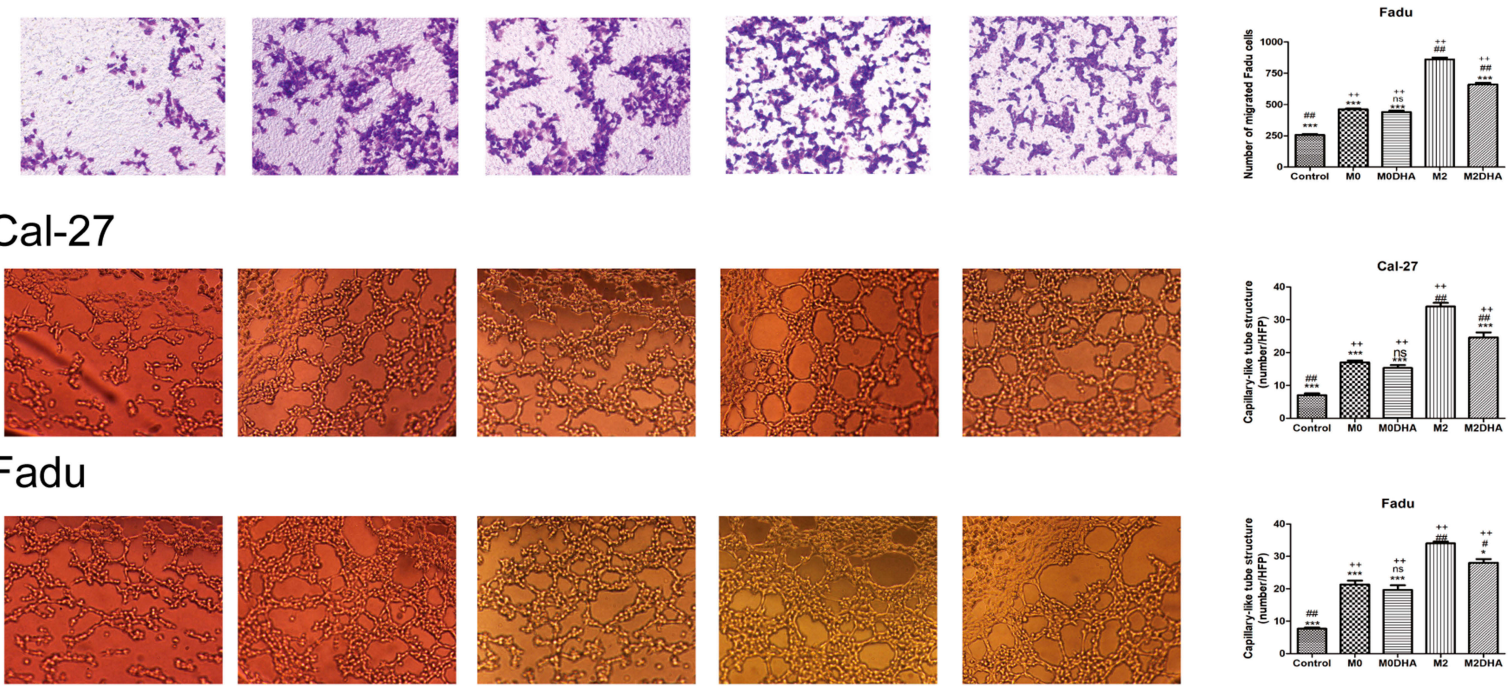

Figure 3 DHA medium inhibited the migration, invasion, and angiogenesis of HNSCC cells. (A) The effect of DHA on HNSCC cells migration was evaluated by woundhealing, and expression of EMT-associated proteins, E-cadherin and Vimentin, was detected by Western blot analysis. (B) The effect of DHA on HNSCC cells invasion was evaluated by transwell assays. (C) The angiogenesis effects of DHA in HNSCC cells were evaluated by tube formation assay.

Notes: Results are present as mean \pm SD. ${ }^{\#} \mathrm{p}<0.0 \mathrm{I}$, \#p <0.05, compared with M0. ${ }^{* * * *} \mathrm{p}<0.0 \mathrm{I},{ }^{*} \mathrm{p}<0.05$, ns $=$ not significant, compared with $\mathrm{M} 2$; ${ }^{++} \mathrm{p}<0.0 \mathrm{I}$, ns $=$ not significant, compared with controls. 
A

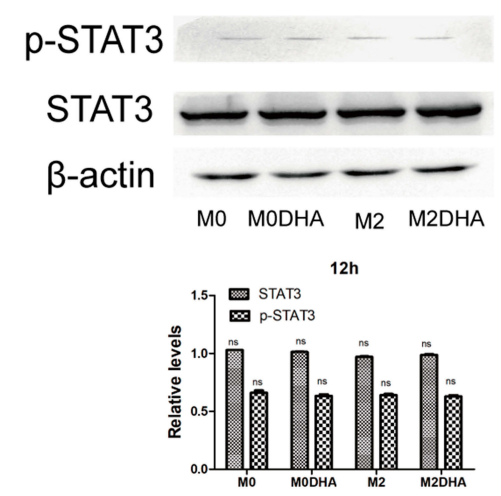

$24 h$

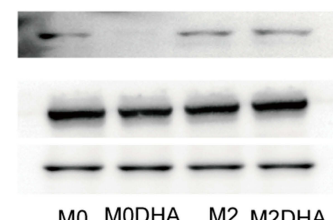

24h

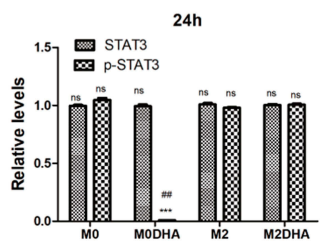

B
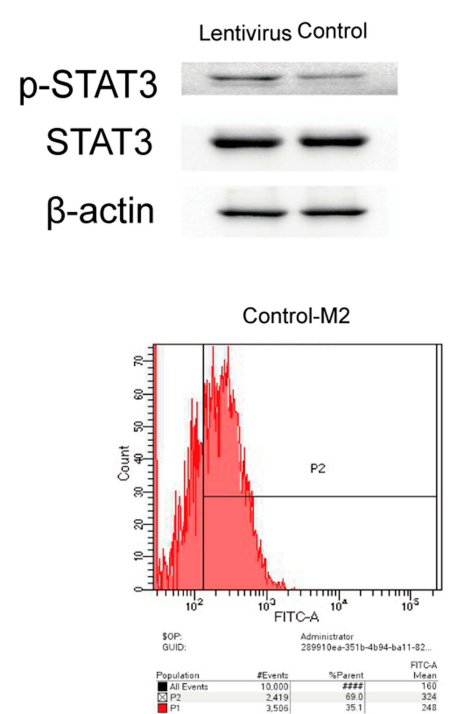

D

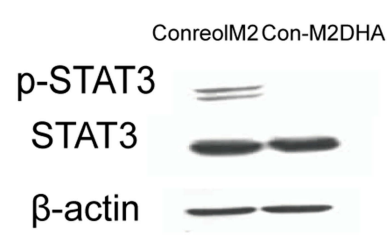

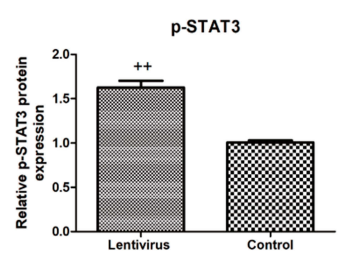

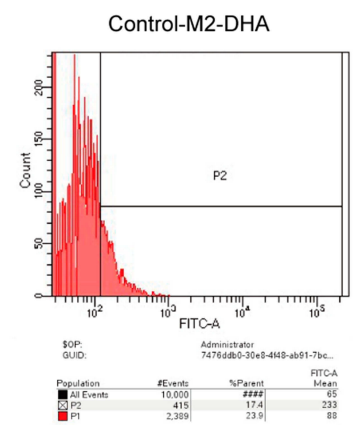

$36 \mathrm{~h}$

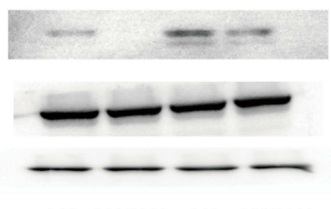

MO MODHA M2 M2DHA

$36 \mathrm{~h}$
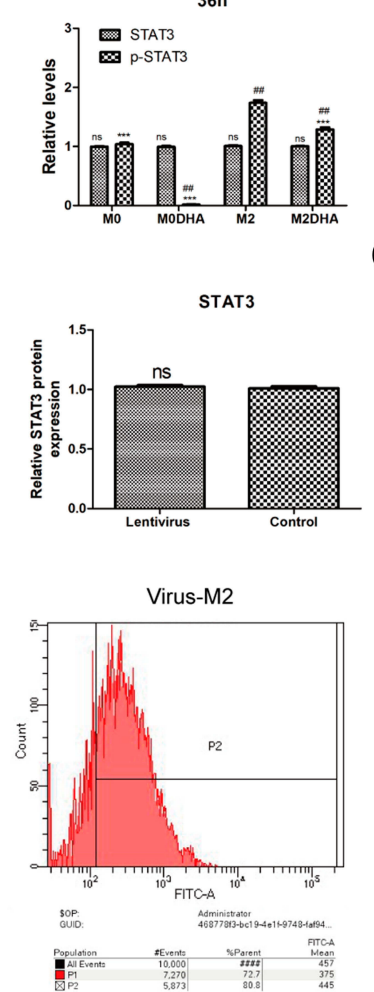

48h

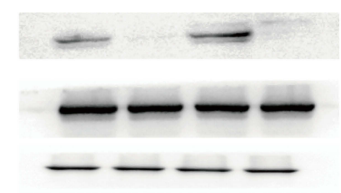

MO MODHA M2 M2DHA

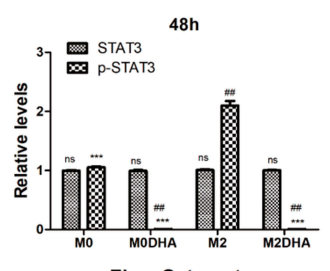

C Flow Cytometry
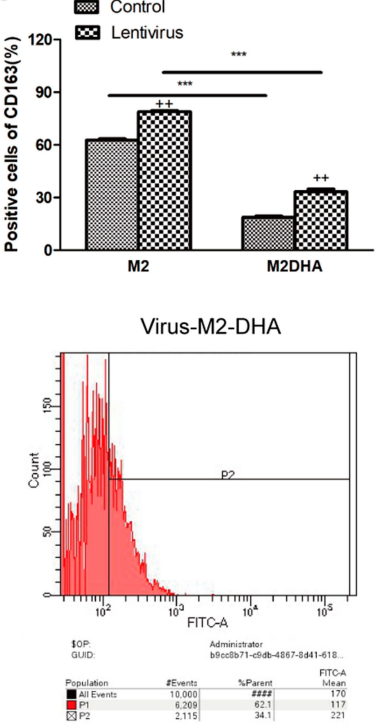

STAT3

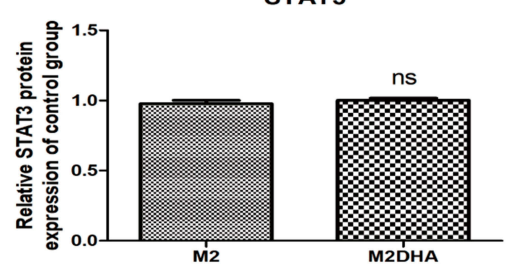

VirusM2 VirusM2DHA
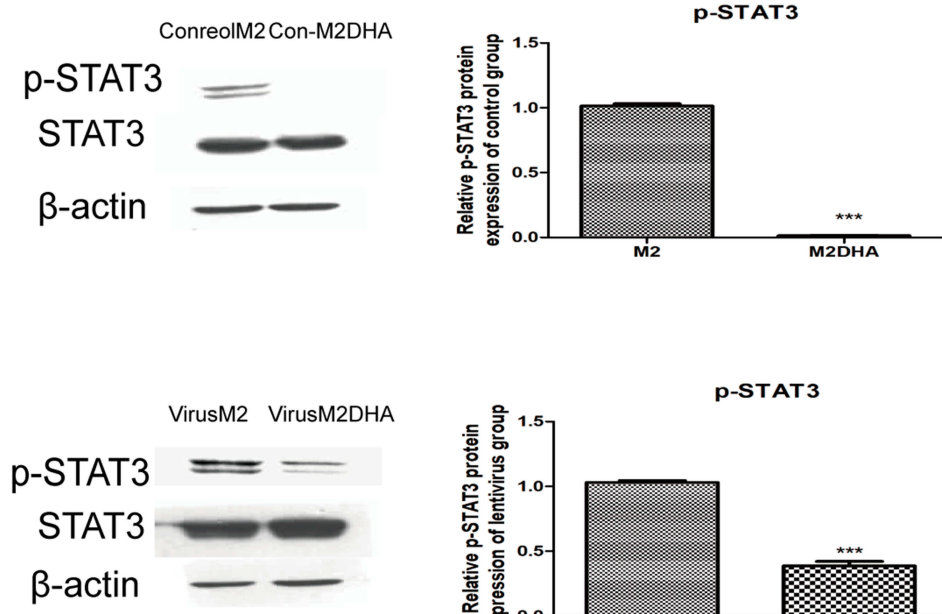

p-STAT 3

STAT3
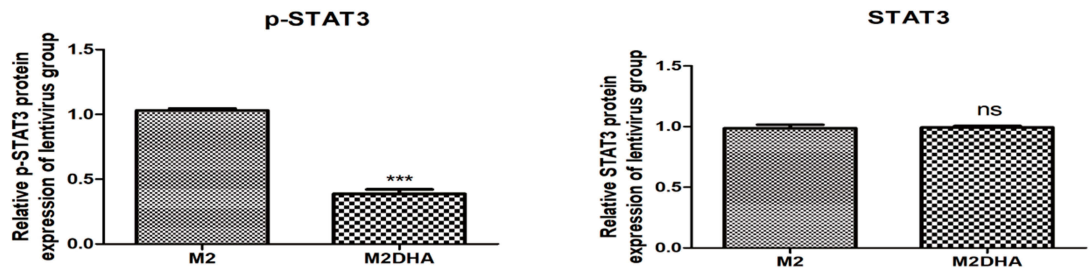

Figure 4 DHA inhibited STAT3 signaling in M2-like TAMs. (A) P-STAT3 and STAT3 protein expression levels during polarization or inhibition of polarization. (B) Macrophages were transfected and p-STAT3 protein expression activated and detected by Western blot. (C) Lentivirus treatment attenuated the inhibitory effects of DHA on M2-like TAMs. (D) Lentiviral treatment prevented complete inhibition of STAT3 phosphorylation by DHA, as determined by Western blot.

Notes: Results are present as mean $\pm \mathrm{SD} .{ }^{\#} \mathrm{p}<0.0 \mathrm{I}$, ns=not significant, compared with $\mathrm{M0}$; ${ }^{* * *} \mathrm{p}<0.0 \mathrm{I}$, ns = not significant, compared with $\mathrm{M} 2$; ${ }^{++} \mathrm{P}<0.0 \mathrm{I}$, ns $=$ not significant, compared with control. 
derivative-12 can regulate the polarization of macrophages and upregulate the number and activity of M1-like macrophages. ${ }^{39}$ Celastrus orbiculatus can reduce the expression of MMP-9 in gliomas, thereby reducing the recruitment of macrophages by primary cancers. ${ }^{40}$ Astragaloside can inhibit the polarization of M2-like macrophages and reduce the proportion of M2-like TAMs by modulating macrophage polarization through adenosine 5'-monophosphate-activated protein kinase (AMPK) signaling. ${ }^{41}$ Apart from these, IL-6 has been proven to promote polarization of M2-like TAMs through activation of STAT3. ${ }^{25,26}$ In view of the fact that DHA could block phosphorylation and thus activation of STAT $3,{ }^{24}$ we turned to test DHA as a potential TAMtargeted agent that is capable of inhibiting the polarization of M2-like TAMs.

Macrophages have various properties that can be altered by changes in the tumor environment. ${ }^{41}$ In many tumors, IL-6 is a highly abundant cytokine in the tumor microenvironment. ${ }^{42-44}$ IL-6 increases the amount of IL-4 receptor (IL-4R) on the cell surface, making macrophages more sensitive to IL-4 and thus more likely to be polarized into M2 macrophages. This pathway is regulated by STAT3 phosphorylation activation via IL-6 receptor. ${ }^{45}$ Il4ra is sensitive to STAT3 stimulation by any STAT3activating receptor, leading to increased IL-4R expression and increased sensitivity to IL-4. Our flow cytometry and qRT-PCR assays showed that IL-4 and IL- 6 can induce polarization of M2 macrophages. These results are consistent with the findings of Chen et al and Fu et al. ${ }^{46,47}$

In the tumor microenvironment, STAT3 play roles in the tumor progression and metastasis. STAT3 is activated in numerous primary tumors and exerts multiple protumor effects by regulating its downstream genes. ${ }^{40-45}$ We found that DHA as a putative STAT3 inhibitor could inhibit HNSCC by blocking STAT3 activation, and thus downregulates a series of its downstream genes including cyclin D1, B-cell lymphoma-xl (Bcl-xL), B-cell lymphoma-1 (Bcl-1), E-cadherin, matrix metalloproteinase-2 (MMP2), MMP9, etc., of which proteins are associated with regulation of cell cycle, apoptosis, and epithelial-mesenchymal transition (EMT). ${ }^{24}$ Wang et al showed that DHA could prevent cancer stem cell (CSCs) metastasis by inhibiting EMT. $^{48}$ In vivo, they found that DHA reduce lung metastasis formation caused by CSC and prolong survival of metastasis-bearing mice, with inhibition of STAT3 activation and downregulation of MMP-9, MMP-2, N-cadherin and upregulate E-cadherin in lung metastatic tumors.
Hence, DHA is an effective drug suppressing migration and invasion of HNSCC. The effect of DHA on M2-like TAM polarization as well as tumor progression deserves to be further elucidated.

In many tumors, activation of macrophage STAT3 promotes M2 macrophage polarization. ${ }^{49-51} \mathrm{We}$ found that levels of p-STAT3 in macrophages increased in a timedependent manner under stimulation with IL-4/IL-6, while the expression of CD163, an M2-like TAM marker, was also increased. When phosphorylation was inhibited, CD163 expression decreased. These results suggest that STAT3 phosphorylation is involved in M2-like TAM polarization. DHA is a semisynthetic derivative of artemisinin, a drug that blocks the phosphorylation of tyrosine residue 705 in the STAT3 protein, which is essential for STAT3 activation. ${ }^{24}$ By transducing macrophages using lentiviruses, we demonstrated that DHA inhibits M2-like macrophage polarization and reduced the number of M2like TAMs by blocking phosphorylation of STAT3 in macrophages. In vitro, STAT3 expression levels were related to the number of macrophages and did not change with increased or decreased M2-like TAM numbers. These results indicate that inactivation of the STAT3 pathway is directly related to the inhibitory effect of DHA on M2-like TAM polarization.

In 1893, Rudolf Virchow proposed a relationship between tumor and inflammation based on his clinical observations; however, the molecular mechanism underlying this association has yet to be fully elucidated. Chronic inflammatory stimulation has always been the basis of tumor formation and is regarded as an epidemiological risk factor for most solid tumors. NF- $\mathrm{KB}$ is one of the most important inflammatory signals, while tumor necrosis factor- $\alpha$ (TNF- $\alpha$ ), interleukin- $1 \beta$ (IL-1 $\beta$ ), and other inflammatory factors can promote the growth and angiogenesis of head and neck cancer, and other cancers, through activation of NF- $\kappa$ B. ${ }^{52,53}$ Huang et al showed that, in an inflammatory environment, DHA inhibits $\mathrm{NF}-\kappa \mathrm{B}$ pathway activation in an Nrf2-dependent manner, thus preventing the production of TNF- $\alpha$ and IL- $1 \beta$ by primary macrophages. ${ }^{54}$ In a study of the parasitic disease malaria, the malarial pigment hemoglobin $(\mathrm{Hz})$, a heme metabolite produced by Plasmodium, was found to induce human M0 macrophages to polarize into M2 macrophages under physiological conditions. ${ }^{55}$ Further, the combination of chloroquine and artemisinin can inhibit M2 macrophage polarization by blocking activation of the NF- $\mathrm{KB}$ and PI3K/Akt pathways. These findings 
suggest that dihydroartemisinin as a derivative of artemisinin could inhibit M2 macrophage polarization by blocking activation of the NF- $\mathrm{KB}$ and PI3K/Akt pathways, which might also be happening in tumor microenvironment.

Noori et al showed that DHA can shift the Th2 immune response toward a Th1 response, significantly reducing the number of CD4(+) $\mathrm{T}$ cells in animal spleen and physiological IL-4 levels in the animal model. ${ }^{56}$ In the breast cancer model, CD4(+) T cells release IL-4 and interleukin-13 (IL-13), and IL-4 induces macrophage polarization into M2-like TAMs via AMPK signaling in the tumor microenvironment. ${ }^{39,57}$ When IL-4 expression is greatly reduced in vivo, its ability to polarize M2 macrophages through the AMPK pathway will inevitably decrease, thereby also reducing M2-like TAM numbers, consistent with our experimental findings. Cho et al showed that artemisinin (also a derivative of Artemisia annua) significantly stimulates M1 macrophage induction by lipopolysaccharide (LPS), as well as interleukin-12 $\beta$ (IL-12 $\beta$ ) production. $^{58}$ Artemisinin stimulates the production of IL-12 $\beta$ by inhibiting JNK signaling pathway activation and IL-12 $\beta$ has an important role in generation of the Th1 immune response, triggering NK cell activation, CD4(+) Th1 cell production, CD8(+) $\mathrm{T}$ cell responses, and other anti-tumor activities. ${ }^{59,60}$ Production of reactive oxygen species (ROS) downstream of LPS signaling in macrophages mediates the production of inflammatory cytokines. Upregulation mitochondrial ROS (mROS) expression is key to the antibacterial activity of M1 macrophages, and ROS production may be important for the activation and function of M1 macrophages. ${ }^{61}$ DHA promotes ROS production in cancer; ${ }^{62}$ therefore, we further addressed whether DHA can induce M2-like TAMs to polarize into M1-type macrophages, which can participate in the Th1 immune response and kill tumor cells.

Cyclooxygenase-2 (COX-2) is an inducible enzyme that catalyzes the synthesis of prostaglandins and a common target for chemotherapy. Abrahão et al and Lin et al demonstrated that COX-2 is involved in proliferation of head and neck cancer cells. The expression of COX-2 is significantly increased in HNSCC. Therefore, COX-2 could be used as a treatment target for HNSCC. ${ }^{63,64} \mathrm{Kim}$ et al reported that dihydroartemisinin can effectively inhibit COX-2 production in macrophages by downregulating PI3K/Akt and MAPK pathway activity. ${ }^{65}$ These findings suggest that dihydroartemisinin could inhibit proliferation of head and neck cancer cells by reducing the expression of COX-2 in M2-like TAMs in tumor microenvironment.

In vitro experiments showed that DHA can inhibit M2like TAM polarization; however, the generation, progression, and metastasis of human tumors is an extended inflammatory process, where the induced inflammatory factors are persistent and promote constant changes in the tumor environment, resulting in macrophages receiving various activation signals over an extended period, during which polarization may occur at any time. Polarization may not occur alone; however, it will be a continuous process. Insoluble inflammatory factors may be present for hours, days, months, or years; however, the accompanying immune response maybe stopped at any time, leading to considerable uncertainty. Macrophage polarization is a dynamic process that involves the balance between various cytokines and chemokines and the recruitment of other immune or cancer cells. Therefore, our experiments can only reflect the changes in this dynamic process at a particular time point or under specific conditions.

In conclusion, our experiments show that DHA can partially block the polarization of M2-like macrophages by inhibiting STAT3 signaling activation in macrophages, thereby reducing the invasion, migration, and angiogenesis of HNSCC. Our study suggests that M2-like TAMs are a potential target for cancer therapy and provide a new sight regarding therapeutic potential of DHA in HNSCC treatment.

\section{Acknowledgments}

This study was supported by the Key Research and Development Project Plan of Hebei Province Science and Technology Department (No. 18277736D), and Key Subject of Medical Science Research in Hebei Province of China (No. 20170201).

\section{Disclosure}

The authors report no conflicts of interest in this work.

\section{References}

1. Sivanantham B, Sethuraman S, Krishnan UM. Combinatorial effects of curcumin with an anti-neoplastic agent on head and neck squamous cell carcinoma through the regulation of EGFR-ERK1/2 and apoptotic signaling pathways. ACS Comb Sci. 2016;18(1):22-35. doi:10.1021/ acscombsci.5b00043

2. Marur A, Forastiere A. Head and neck cancer: changing epidemiology, diagnosis, and treatment. Mayo Clin Proc. 2008;83(4):489-501. doi: $10.4065 / 83.4 .489$ 
3. Salo T, Vered M, Bello IO, et al. Insights into the role of components of the tumor microenvironment in oral carcinoma call for new therapeutic approaches. Exp Cell Res. 2014;325(2):58-64. doi:10.1016/j. yexcr.2013.12.029

4. Marx V. Tracking metastasis and tricking cancer. Nature. 2013;494 (7435):133-136. doi:10.1038/494131a

5. Noy R, Pollard JW. Tumor-associated macrophages: from mechanisms to therapy. Immunity. 2014;41(1):49-61. doi:10.1016/j. immuni.2014.06.010

6. Robinson BD, Sica GL, Liu YF, et al. The tumor microenvironment of metastasis in human breast carcinoma. A potential prognostic marker linked to hematogenous dissemination. J Clin Cancer Res. 2005;15(7):2433-2441. doi:10.1158/1078-0432.CCR-08-2179

7. Mantovani A, Sozzani S, Locati M, et al. Macrophage polarization: tumor-associated macrophages as a paradigm for polarized M2 mononuclear phagocytes. Trends Immunol. 2002;23(11):549-555. doi:10.1016/S1471-4906(02)02302-5

8. Han Q, Shi H, Liu F. CD163 M2-type tumor-associated macrophage support the suppression of tumor-infiltrating $\mathrm{T}$ cells in osteosarcoma. Int Immunopharmacol. 2016;34:101-106. doi:10.1016/j.intimp.2016.01.023

9. Kimura Y, Sumiyoshi M, Baba K. Antitumor and antimetastatic activity of synthetic hydroxystilbenes through inhibition of lymphangiogenesis and M2 macrophage differentiation of tumor-associated macrophages. Anticancer Res. 2016;36(1):137-148.

10. Jeong SK, Kim JS, Lee CG, et al. Tumor associated macrophages provide the survival resistance of tumor cells to hypoxic microenvironmental condition through IL-6 receptor-mediated signals. Immunobiology. 2017;222(1):55-65. doi:10.1016/j.imbio.2015. 11.010

11. Hu Y, He MY, Zhu LF, et al. Tumor-associated macrophages correlate with the clinicopathological features and poor outcomes via inducing epithelial to mesenchymal transition in oral squamous cell carcinoma. J Exp Clin Cancer Res. 2016;35(1):12. doi:10.1186/ s13046-015-0281-z

12. Lu-Emerson C, Snuderl M, Kirkpatrick ND, et al. Increase in tumor-associated macrophages after antiangiogenic therapy is associated with poor survival among patients with recurrent glioblastoma. Neuro Oncol. 2013;15(8):1079-1087. doi:10.1093/neuonc/not082

13. He KF, Zhang L, Huang CF, et al. CD163+ tumor-associated macrophages correlated with poor prognosis and cancer stem cells in oral squamous cell carcinoma. Biomed Res Int. 2014;2014:838632. doi: $10.1155 / 2014 / 838632$

14. Gutman J, Kovacs S, Dorsey G, et al. Safety, tolerability, and efficacy of repeated dose of dihydroartemisinin-piperaquine for prevention and treatment of malaria: a systematic review and meta-analysis. Lancet Infect Dis. 2017;17(2):184-193. doi:10.1016/S14733099(16)30378-4

15. Wu C, Liu J, Pan X, et al. Design, synthesis and evaluation of the antibacterial enhancement activities of amino dihydroartemisinin derivatives. Molecules. 2013;18(6):6866-6882. doi:10.3390/ molecules 18066866

16. Zhang CZ, Zhang H, Yun J, et al. Dihydroartemisinin exhibits antitumour activity toward hepatocellular carcinoma in vitro and in vivo. Biochem Pharmacol. 2012;83(9):1278-1289. doi:10.1016/j. bcp.2012.02.002

17. Jiao Y, Ge CM, Meng QH, et al. Dihydroartemisinin is an inhibitor of ovarian cancer cell growth. Acta Pharmacol Sin. 2007;28 (7):1045-1056. doi:10.1111/j.1745-7254.2007.00612.x

18. Sica A, Mantovani A. Macrophage plasticity and polarization: in vivo veritas. J Clin Invest. 2012;122(3):787-795. doi:10.1172/JCI59643

19. Minam K, Hiwatashi K, Ueno S, et al. Prognostic significance of CD68, CD163 and folate receptor- $\beta$ positive macrophages in hepatocellular carcinoma. Exp Ther Med. 2018;15(5):4465-4476. doi:10.3892/etm.2018.5959
20. Lao L, Fan S, Son E. Tumor associated macrophages as therapeutic targets for breast cancer. Adv Exp Med Biol. 2017;1026:331-370. doi:10.1007/978-981-10-6020-5_16

21. Chen S, Zheng P, Wang W, et al. Aberrant expression of NOR1 protein in tumor associated macrophages contributes to the development of DEN-induced hepatocellular carcinoma. J Cell Physiol. 2018;233(6):5002-5013. doi:10.1002/jcp.26349

22. Krishnan V, Schaar B, Tallapragada S, et al. Tumor associated macrophages in gynecologic cancers. Gynecol Oncol. 2018;149 (1):205-213. doi:10.1016/j.ygyno.2018.01.014

23. LWang ZG, Si XL, Xu A, et al. Activation of STAT3 in human gastric cancer cells via interleukin-6-type cytokine signaling correlates with clinical implications. PLoS One. 2013;8(10):e75788. doi:10.1371/journal.pone.0075788

24. Jia LF, Zhou CY, Li MX, et al. Dihydroartemisinin as a putative STAT3 inhibitor, suppresses the growth of head and neck squamous cell carcinoma by targeting Jak2/STAT3 signaling. PLoS One. 2016;19:1-17. doi:10.1371/journal.pone.0147157

25. Yoshiyuki K, Maho S. Resveratrol prevents tumor growth and metastasis by inhibiting lymphangiogenesis and M2 macrophage activation and differentiation in tumor-associated macrophages. Nutr Cancer. 2016;1-12.

26. Ragione FD, Cucciolla V, Borriello A, et al. Resveratrol arrests the cell division cycle at $\mathrm{S} / \mathrm{G} 2$ phase transition. Biochem Biophys Res Commun. 1998;250(1):53-58. doi:10.1006/bbrc.1998.9263

27. El-Rouby DH. Association of macrophages with angiogenesis in oral verrucous and squamous cell carcinomas. J Oral Pathol Med. 2010;39(7):559-564. doi:10.1111/j.1600-0714.2010.00879.x

28. Salvesen HB, Akslen LA. Significance of tumour-associated macrophages, vascular endothelial growth factor and thrombospondin-1 expression for tumour angiogenesis and prognosis in endometrial carcinomas. Int J Cancer. 1999;84(5):539-543. doi:10.1002/(SICI) 1097-0215(19991022)84:5<538::AID-IJC17>3.0.CO;2-B

29. Migita T, Sato E, Saito K, et al. Differing expression of MMPs-1 and 9 and urokinase receptor between diffuse and intestinal type gastric carcinoma. Int J Cancer. 1999;84(1):74-79. doi:10.1002/(SICI)10970215(19990219)84:1<74::AID-IJC14>3.0.CO;2-I

30. Koukourakis MI, Giatromanolaki A, Kakolyris S, et al. Different patterns of stromal and cancer cell thymidine phosphorylase reactivity in non-small-cell lung cancer: impact on tumour neo-angiogenesis and survival. Br J Cancer. 1998;77:1696-1703.

31. Zhang QW, Liu L, Gong CY, et al. Prognostic significance of tumorassociated macrophages in solid tumor: A meta-analysis of the literature. PLoS One. 2012;7(12):e50946. doi:10.1371/journal.pone.0050946

32. Weber M, Moebius P, Büttner-Herold M, et al. Macrophage polarisation changes within the time between diagnostic biopsy and tumour resection in oral squamous cell carcinomas - an immunohistochemical study. $\mathrm{Br}$ J Cancer. 2015;113(3):510-519. doi:10.1038/bjc.2015.212

33. Weber M, Iliopoulos C, Moebius P, et al. Prognostic significance of macrophage polarization in early stage oral squamous cell carcinomas. Oral Oncol. 2016;52:75-84. doi:10.1016/j.oraloncology.2015.11.001

34. Marcus B, Arenberg D, Lee J, et al. Prognostic factors in oral cavity and oropharyngeal squamous cell carcinoma. Cancer. 2004;101 (12):2779-2787. doi:10.1002/cncr.20701

35. Okubo M, Kioi M, Nakashima H, et al. M2-polarized macrophages contribute to neovasculogenesis, leading to relapse of oral cancer following radiation. Sci Rep. 2016;6(1):27548. doi:10.1038/srep27548

36. Mori K, Hiroi M, Shimada J, Ohmori Y. Infiltration of M2 tumor associated macrophages in oral squamous cell carcinoma correlates with tumor malignancy. Cancers. 2011;3(4):3726-3739. doi:10.3390/ cancers 3043726

37. Mrad M, Imbert C, Garcia V, et al. Downregulation of sphingosine kinase-1 induces protective tumor immunity by promoting M1 macrophage response in melanoma. Oncotarget. 2016;7(44):71873-71886. doi:10.18632/oncotarget.12380 
38. Sugimura K, Miyata H, Tanaka K, et al. High infiltration of tumorassociated macrophages is associated with a poor response to chemotherapy and poor prognosis of patients undergoing neoadjuvant chemotherapy for esophageal cancer. J Surg Oncol. 2015;111 (6):752-759. doi:10.1002/jso.23881

39. Kong LN, Lin H, Huang $\mathrm{C}$, et al. Hesperetin derivative-12 (HDND-12) regulates macrophage polarization by modulating JAK2/STAT3 signaling pathway. Chin J Nat Med. 2019;17 (2):0122-0130. doi:10.1016/S1875-5364(19)30014-7

40. Gu H, Wang HB, Feng J, et al. Celastrus orbiculatus extract inhibits the migration and invasion of human glioblastoma cells in vitro. BMC Complement Altern Med. 2016;16(1):387. doi:10.1186/s12906-016-1232-8

41. Xu F, Cui WQ, Wei Y, et al. Astragaloside IV inhibits lung cancer progression and metastasis by modulating macrophage polarization through AMPK signaling. J Exp Clin Cancer Res. 2018;37(1):207. doi:10.1186/s13046-018-0878-0

42. Azare J, Doane A, Leslie K, et al. Stat3 mediates expression of autotaxin in breast cancer. PLoS One. 2011;6(11):e27851. doi:10.1371/journal.pone.0027851

43. Xiong $\mathrm{H}$, Zhang ZG, Tian XQ, et al. Inhibition of JAK1, 2/STAT3 signaling induces apoptosis, cell cycle arrest, and reduces tumor cell invasion in colorectal cancer cells. Neoplasia. 2008;10(3):287-297. doi:10.1593/neo.07971

44. Leeman RJ, Lui VWY, Grandis JR. STAT3 as a therapeutic target in head and neck cancer. Expert Opin Biol Ther. 2006;6(3):231-241. doi:10.1517/14712598.6.3.231

45. Mauer J, Chaurasia B, Goldau J, et al. Signaling by IL-6 promotes alternative activation of macrophages to limit endotoxemia and obesity-associated resistance to insulin. Nat Immunol. 2014;15 (5):423-430. doi:10.1038/ni.2865

46. Chen L, Wang S, Wang Y, et al. IL-6 influences the polarization of macrophages and the formation and growth of colorectal tumor. Oncotarget. 2018;9(25):17443-17454. doi:10.18632/oncotarget.24734

47. Fu XL, Duan W, Su CY, et al. Interleukin 6 induces M2 macrophage differentiation by STAT3 activation that correlates with gastric cancer progression. Cancer Immunol Immunother. 2017;16(12):1597-1608. doi:10.1007/s00262-017-2052-5

48. Wang W, Sun Y, Li X, et al. Dihydroartemisinin prevents distant metastasis of laryngeal carcinoma by inactivating STAT3 in cancer stem cells. Med Sci Monit. 2020;26:e9223483. doi:10.12659/MSM.922348

49. Yuan FJ, Fu X, Shi HF, et al. Induction of murine macrophage M2 polarization by cigarette smoke extract via the JAK2/STAT3 Pathway. PLoS One. 2014;9(9):e107063. doi:10.1371/journal.pone.0107063

50. Hasita H, Komohara Y, Okabe H, et al. Significance of alternatively activated macrophages in patients with intrahepatic cholangiocarcinoma. Cancer Sci. 2010;101(8):1913-1919. doi:10.1111/j.1349-7006.2010.01614.x

51. Shiraishi D, Fujiwara Y, Komohara Y, et al. Glucagon-like peptide-1 (GLP-1) induces M2 polarization of human macrophages via STAT3 activation. Biochem Biophys Res Commun. 2012;425(2):304-308. doi:10.1016/j.bbrc.2012.07.086

52. Liu YP, Lee JJ, Lai TC, et al. Suppressive function of low-dose deguelin on the invasion of oral cancer cells by downregulating tumor necrosis factor alpha-induced nuclear factor-kappa B signaling. Head Neck. 2016;38(1):E524-E534. doi:10.1002/ hed. 24034

OncoTargets and Therapy

\section{Publish your work in this journal}

OncoTargets and Therapy is an international, peer-reviewed, open access journal focusing on the pathological basis of all cancers, potential targets for therapy and treatment protocols employed to improve the management of cancer patients. The journal also focuses on the impact of management programs and new therapeutic

Submit your manuscript here: https://www.dovepress.com/oncotargets-and-therapy-journa
53. Koo JS, Kim YD, Jetten AM, et al. Overexpression of mucin genes induced by interleukin-1 beta, tumor necrosis factor-alpha, lipopolysaccharide, and neutrophil elastase is inhibited by a retinoic acid receptor alpha antagonist. Exp Lung Res. 2012;28(4):315-332. doi:10.1080/01902140252964393

54. Huang XT, Liu W, Zhou Y, et al. Dihydroartemisinin attenuates lipopolysaccharide-induced acute lung injury in mice by suppressing $\mathrm{NF}-\kappa \mathrm{B}$ signaling in an Nrf2-dependent manner. Int $\mathrm{J}$ Mol Med. 2019;44(6):2213-2222. doi:10.3892/ijmm.2019.4387

55. Bobade D, Khandare AV, Deval M, et al. Hemozoin-induced activation of human monocytes toward M2-like phenotype is partially reversed by antimalarial drugs chloroquine and artemisinin. Microbiologyopen. 2019;8(3):e00651. doi:10.1002/mbo3.651

56. Noori S, Hassan ZM. Dihydroartemisinin shift the immune response towards Th1, inhibit the tumor growth in vitro and in vivo. Cell Immunol. 2011;271(1):67-72. doi:10.1016/j.cellimm.2011.06.008

57. DeNardo DG, Barreto JB, Andreu P, et al. CD4(+) T cells regulate pulmonary metastasis of mammary carcinomas by enhancing protumor properties of macrophages. Cancer Cell. 2009;16(2):91-102. doi:10.1016/j.ccr.2009.06.018

58. Cho CY, Lee SH, Lee M, et al. Enhanced IL-12p40 production in LPS-stimulated macrophages by inhibiting JNK activation by artemisinin. Arch Pharm Res. 2012;35(11):1961-1968. doi:10.1007/ s12272-012-1113-8

59. Kobayashi M, Kweon MH, Kuwata H, et al. Toll-like receptor-dependent production of IL-12p40 causes chronic enterocolitis in myeloid cell-specific Stat3-deficient mice. J Clin Invest. 2003;111(9):1297-1308. doi:10.1172/JCI17085

60. Watkins SK, Egilmez NK, Suttles J, et al. IL-12 rapidly alters the functional profile of tumor-associated and tumor-infiltrating macrophages in vitro and in vivo. J Immunol. 2007;178(3):1357-1362. doi:10.4049/jimmunol.178.3.1357

61. Covarrubias A, Byles V, Horng T. ROS sets the stage for macrophage differentiation. Cell Res. 2013;23(8):984-985. doi:10.1038/ cr.2013.88

62. Hu W, Chen SS, Zhang JL, et al. Dihydroartemisinin induces autophagy by suppressing NF-kB activation. Cancer Lett. 2014;343 (2):239-248. doi:10.1016/j.canlet.2013.09.035

63. Abrahão AC, Giudice FS, Sperandio FF, et al. Effects of celecoxib treatment over the AKT pathway in head and neck squamous cell carcinoma. J Oral Pathol Med. 2013;42(10):793-798. doi:10.1111/ jop. 12081

64. Lin DT, Subbaramaiah K, Shah JP, et al. Cyclooxygenase-2: a novel molecular target for the prevention and treatment of head and neck cancer. Head Neck. 2002;24(8):792-799. doi:10.1002/hed.10108

65. Kim HG, Yang JH, Han EH, et al. Inhibitory effect of dihydroartemisinin against phorbol ester-induced cyclooxygenase-2 expression in macrophages. Food Chem Toxicol. 2013;56:93-99. doi:10.1016/j. fct.2013.02.017

agents and protocols on patient perspectives such as quality of life, adherence and satisfaction. The manuscript management system is completely online and includes a very quick and fair peer-review system, which is all easy to use. Visit http://www.dovepress.com/ testimonials.php to read real quotes from published authors. 